\title{
Differential white cell counts by frequency distribution analysis of cell volumes
}

\author{
N. C. HUGHES-JONES, IAN NORLEY, JANET M. S. YOUNG, AND \\ J. M. ENGLAND
}

From the Medical Research Council Experimental Haematology Unit, St Mary's Hospital Medical School, London

SYNOPSIS Absolute neutrophil and lymphocyte counts on peripheral blood can be made by analysis of the output from a Coulter particle counter, utilizing the difference in the relative cell volume between these two types of cell. A comparison has been made between the results obtained by volume analysis and those obtained by standard microscopical techniques in 10 normal people and 45 patients. The absolute neutrophil count obtained by volume analysis agreed well with values obtained by microscopy; the lymphocyte count did not give such good agreement, since the smaller number of cells counted gave rise to larger sampling errors. The method of volume analysis is suitable for the assessment of absolute neutrophil counts for clinical use.

Polymorphonuclear neutrophils have approximately twice the cellular volume of lymphocytes and the two populations can be differentiated by pulseheight analysis of the output from a Coulter particle counter (Van Dilla, Fulwyler, and Boone, 1967). It has been suggested that this method of analysis could be used for carrying out differential white cell counts (Gauthier and Harel, 1967; Oberjat, Zucker, and Cassen, 1970), and it has been possible to obtain a good correlation between the results of differential counts obtained by examination of stained blood films and those obtained by volume analysis in normal people and in selected patients (Humphries and Miller, 1972). We have assessed the feasibility of using volume analysis in a routine haematology laboratory by carrying out differential counts by this method on 45 patients in whom the clinician had requested the investigation.

\section{Methods}

DILUTION OF WHOLE BLOOD AND LYSIS OF RED CELLS

Whole blood $(0 \cdot 1 \mathrm{ml})$ was added to $20 \mathrm{ml}$ of Isoton (Coulter Electronics) and followed by $0.1 \mathrm{ml}$ of saponin solution. The saponin solution was prepared from saponin powder (Coulter Electronics): the concentration of the stock saponin solution was approximately $1 \mathrm{~g} / 100 \mathrm{ml}$ but the precise concentra-

Received for publication 6 May 1974. tion required was different for each batch of saponin and was adjusted so that visual lysis of the red cells started after about one min and was complete in about 1.5 minutes. Three min after the addition of saponin, $1 \mathrm{ml}$ of filtered $\mathrm{AB}$ serum was added in order to neutralize the activity of the saponin on leucocyte membranes. Frequency distribution curves were determined within the following 10 minutes.

\section{FREQUENCY DISTRIBUTION CURVES OF \\ LEUCOCYTE VOLUMES}

These were obtained using a Coulter model FN particle counter and a Coulter pulse-height analyzer (model C 1000). Counting was continued until 1000 cells had accumulated in the modal channel of the neutrophil peak; this usually took less than one minute.

Blood samples from 10 normal people and from 45 patients sent for routine analysis were investigated within six hr of venepuncture. Neutral EDTA was used as an anticoagulant.

TOTAL WHITE CELL COUNTS

The total white cell counts were obtained from the Coulter model S particle counter.

DIFFERENTIAL WHITE CELL COUNTS

Differential white cell counts were also obtained by standard visual methods from blood films stained with May-Grünwald-Giemsa. A total of 500 cells were counted on films made from normal subjects and 100 cells on films made from patients. 


\section{Results}

EFFECT OF SAPONIN ON THE FREQUENCY DISTRIBUTION CURVES OF LEUCOCYTE VOLUMES

The optimum amount of saponin was found to be that which brought about lysis starting about one minute after its addition and was visually complete in 1.5 minutes. Less saponin left too many red cells intact, and a greater concentration brought about rapid changes in the volume of both lymphocytes and neutrophils. If serum was not added to the white cell suspension three min after the addition of saponin, there was a slow but continuous volume change in the leucocytes, manifesting as a gradual reduction in the height of the lymphocyte and neutrophil peaks, and the appearance of a new peak of small-volumed particles, presumably bare nuclei. The addition of serum partially stabilized the leucocyte volumes, so that no obvious change was detectable for the following 10-15 min, and frequently for longer. The inhibiting action of serum is probably due to its cholesterol content, which reacts with saponin.

Some alteration in the frequency distribution curves of the leucocytes was brought about by saponin even during the initial three-minute period before serum was added. This alteration was shown by comparing curves obtained from saponin-treated cells with those obtained from white cell-rich plasma after allowing the red cells to sediment, when the addition of saponin is not necessary. Saponin slightly increased the modal value of the neutrophil peak and decreased that of the lymphocyte peak and also broadened the curves, indicating an increased variation in size.

\section{IDENTIFICATION OF LYMPHOCYTE AND}

NEUTROPHIL PEAKS

Preparations in which almost all the cells were lymphocytes were obtained either from normal blood (Böyum, 1968) or from patients with chronic lymphocytic leukaemia. Blood from patients with a neutrophil leucocytosis was used for identification of the neutrophil peak. The frequency distribution curves obtained from a relatively pure preparation of lymphocytes and from a normal blood are shown in figure 1. It can be seen that the lymphocyte and neutrophil peaks overlap, as has been found previously (Van Dilla et al, 1967). The neutrophil peak is a composite peak containing eosinophils and monocytes as well as neutrophils. Eosinophils have the same volume as neutrophils, since we found that a patient with an eosinophilia of $8000 / \mu l$ gave a 'neutrophil' peak indistinguishable from those given by normal bloods. Monocytes have a volume slightly

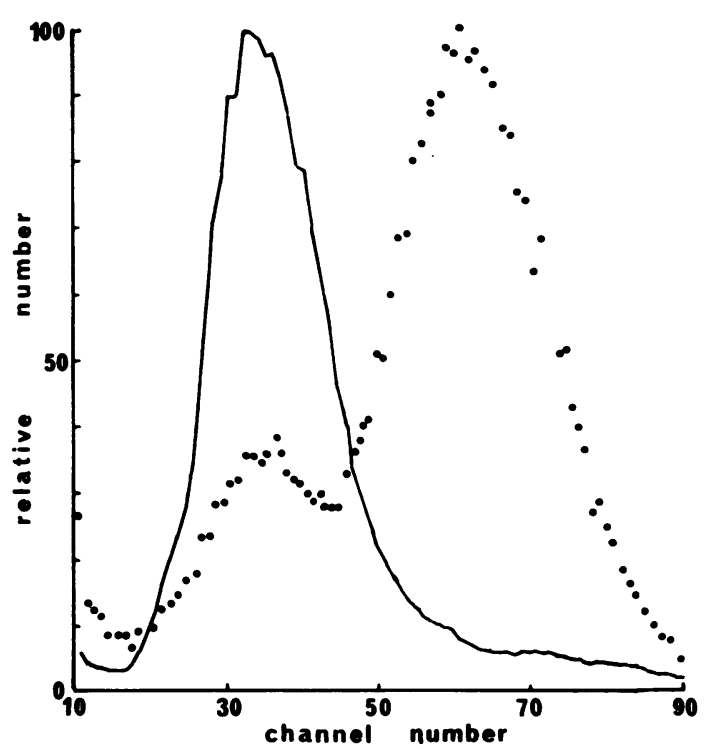

Fig 1 Frequency distribution curves obtained from a relatively pure preparation of lymphocytes (continuous line) and from a normal person (dotted line)

larger than neutrophils (Van Dilla et al, 1967) but since their numbers are only $5-10 \%$ of that of neutrophils, they do not appear as a separate peak but are hidden at the upper end of the neutrophil distribution curve.

THE ANALYSIS OF FREQUENCY DISTRIBUTION CURVES

The analysis of relatively pure preparations of neutrophils and lymphocytes showed that both frequency distribution curves were more nearly described as lognormal than normal. The relative number of cells comprising each peak was therefore estimated by calculating the area under each peak by computer analysis, assuming a lognormal distribution of both peaks in all cases. The absolute number of lymphocytes and neutrophils was then calculated using the total white cell count obtained from the Coulter model S. Comparisons between the absolute neutrophil counts obtained by pulse-height analysis with those obtained by standard visual methods in both normals and patients are shown in figure 2. It can be seen that there is good correlation between the values obtained for the neutrophil counts by the two methods. The ratio of the neutrophil count obtained by pulse-height analysis to that obtained by the visual method ranged from 0.79 to 1.34 in each individual case, the average of all the ratios being 1.05 . 


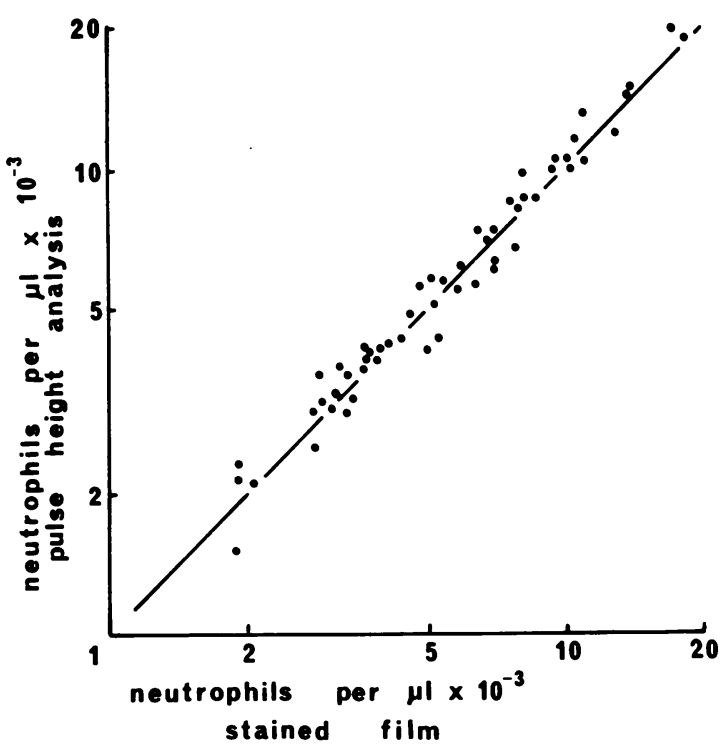

Fig 2 The relationship between the absolute neutrophil counts obtained by standard visual methods from a stained slide and the results obtained by pulse-height analysis

The values for the lymphocyte counts obtained by the two methods did not give as good a correlation, since the smaller number of cells counted gave rise to larger sampling errors. Most values for the ratio of the lymphocyte count obtained by pulseheight analysis to the lymphocyte count obtained by the visual method fell within the range $0 \cdot 6-1 \cdot 5$, but a few fell outside this range, the extremes being $0 \cdot 2$ and $4 \cdot 4$.

\section{Discussion}

An analysis of differential counts carried out in the routine haematology laboratory at St Mary's Hospital showed that in approximately $85 \%$ of cases the clinician primarily wanted to know the absolute neutrophil count. The results presented here demonstrate that an absolute neutrophil count can be quickly estimated by pulse-height analysis of the data taken from the output of a Coulter particle counter.

The neutrophil count was, on average, $5 \%$ higher by pulse-height analysis than by standard visual methods. Although this difference is not statistically significant, such a result might reflect a true difference, since both monocytes and eosinophils are included within the neutrophil curve. In clinical practice, this small overestimation should not lead to error in the interpretation of the count.

The limitation of this technique is that the analysis of the curves is only valid if neutrophils and lymphocytes are the predominant cells. If immature white cells are present or if there is an increase in eosinophils then interpretation of the frequency distribution curves is not possible; it is thus necessary to examine blood films microscopically to exclude both the presence of abnormal nucleated cells and a raised eosinophil count.

Saponin is an impure product containing several different lytic agents and only a few of the preparations examined were found to be suitable for white cell differential counts. It is necessary to use a batch of saponin which lyses red cells rapidly and white cells only slowly; most batches of saponin were found to lyse red cells and white cells equally well. No source of saponin has yet been found which lyses red cells only, but it is possible that one of the lytic agents isolated in pure form might be found to have the required properties.

Although computer analysis of the curves was used in this study, simple methods of analysis can be devised. For instance, it has proved possible to analyse the curves automatically using simple analogue integrating circuits and this is now being assessed. Alternatively, a nomogram can be designed which is based on the relative peak heights of the lymphocytes and neutrophils.

Each differential count takes about five min, but the method is adaptable to a batch technique and it is possible to carry out about 20 estimates in an hour.

\section{References}

Böyum, A. (1968). Isolation of leucocytes from human blood. Further observations. Methylcellulose, dextran and ficoll as erythrocyteaggregating agents. Scand. J. clin. lab. Invest., 21, Suppl., 97, 31-50.

Gauthier, J., and Harel, P. (1967). Human leucocytes: their size distribution and mean corpuscular volume. Canad. med. Ass. J., 97, 793-796.

Humphries, R. K., and Miller, R. G. (1972). Volume analysis of human peripheral blood leukocytes. Ser. Hemat., 5, 142-162.

Oberjat, T. E., Zucker, R. M., and Cassen, B. (1970). Rapid and reliable differential counts on dilute leucocyte suspensions. J. Lab. clin. Med., 76, 518-522.

Van Dilla, M. A., Fulwyler, M. J., and Boone, I. V. (1967). Volume distribution and separation of normal human leucocytes. Proc. Soc. exp. Biol. (N.Y.), 125, 367-370. 